\title{
Checking the Alleged "Incompatibility of the Relic Dark Energy Hypothesis" with Physically Admissible Solutions to the Cosmic Ray Problem of Doubly Special Relativity
}

\author{
Andrew Walcott Beckwith \\ Department of Physics, College of Physics, Chongqing University, Chongqing, China \\ Email: Rwill9955b@gmail.com
}

How to cite this paper: Beckwith, A.W. (2017) Checking the Alleged "Incompatibility of the Relic Dark Energy Hypothesis" with Physically Admissible Solutions to the Cosmic Ray Problem of Doubly Special Relativity. Journal of High Energy Physics, Gravitation and Cosmology, 3, 588-599.

https://doi.org/10.4236/jhepgc.2017.34045

Received: May 8, 2017

Accepted: August 30, 2017

Published: September 2, 2017

Copyright $\odot 2017$ by author and Scientific Research Publishing Inc. This work is licensed under the Creative Commons Attribution International License (CC BY 4.0).

http://creativecommons.org/licenses/by/4.0/

\begin{abstract}
We offer evidence that the Trans Plankian hypothesis about Dark energy is incompatible with necessary and sufficient conditions for solving the cosmic ray problem along the lines presented by Magueijo et al. We can obtain conditions for a dispersion relationship congruent with the Trans Planckian hypothesis only if we cease trying to match cosmic ray data which is important in investigating Doubly Special Relativity. This leads us to conclude that the Trans Planckian hypothesis is inconsistent with respect to current astrophysical data when modeled by Doubly Special Relativity and needs to be seriously revised. Or the Doubly Special Relativity Hypothesis needs to be abandoned.
\end{abstract}

\section{Keywords}

Doubly Special Relativity, Trans Planckian Physics

\section{Introduction}

We examine if an alteration of special relativity presented by Magueijo and Smolin [1], assuming joining the speed of light and Planck energy as a new invariant permits a dispersion relationship which will set dark energy [2] from the "tail mode" of ultra high momentum contributions (of the universe) markedly lower than the total energy of the universe. We find that the answer is yes after modifying an energy equation of $E=M C^{2}$ to obtain a highly non linear dispersion relationship. However, this dispersion relationship does NOT solve the cosmic 
ray problem for low momentum values [1]. Our derived dispersion relationship $\omega_{M}(k)$ matches the Epstein function used by Mercini et al. [2] only if we cease trying to fit cosmic ray data [5] which lead to Magueijo [3] proposing their alteration of special relativity in the first place. We follow Mersini et al. [2] in their derivation of a Trans Planckian dark energy over total energy ratio. Our results argue that we cannot reconcile the requirements of a solution of the "cosmic ray" problem of special relativity in a manner congruent with Mercinis [2] ratios of dark matter energy to total energy being calculated via a Bogoliubov coefficient [4]. The dispersion relationship which we obtained which actually permitted us to calculate the energy of the tail modes of Trans Plankian dark energy [2] vs. total energy ratio [2] to have a value less than ten to the minus 30 power mimics the Epstein function [2] in a manner which contravenes necessary and sufficient conditions [1] for solving the cosmic ray problem of special relativity. Our calculations imply that a Trans-Planckian dark energy depends upon initial conditions which are too specialized and which do not match up with known astrophysical data obtained as of the 1990s. This is in tandem with Lemoine, Martin, and Uzan [5] who dispute on the Trans Planckian hypothesis on different grounds.

\section{Description of Procedure Used to Obtain Energy Density Ratio}

What Mersini [2] did was to use ultra low dispersion relationship values for ultra high momentum values to obtain "ultra low" energy values which were and remain allegedly "frozen" today [2]. They found, using the Epstein function for frequency dispersion relationships a range of frequencies $\leq H_{0}$, where $H_{0}$ is the present Hubble rate of expansion. From there, they computed Trans-Planckian dark energy modes which are about 122 to 123 orders of magnitude smaller than the total energy of the universe assumed for their expansion model. Note in this discussion that $\omega_{K}(k)$ refers to the dispersion relationship Mercini [2] derived, while $\omega_{M}(k)$ will be a dispersion relationship derived from Magueijo and Smolin's [3] modification of special relativity. Mersini [2] changed a standard linear dispersion relationship to one which has a modified Epstein function with a peak value for frequency given when $k=k_{\mathrm{C}}$ and where we have if we can set $k \ll k_{C}$

$$
\omega_{K}^{2}(k) \approx k^{2}
$$

which means for low values of momentum we have a linear relationship for dispersion vs. "momentum" in low momentum situations. In addition we also have that

$$
\omega_{K}^{2}\left(k \gg k_{C}\right) \approx \exp \left(-k / k_{C}\right) \underset{k \rightarrow \infty}{\longrightarrow} 0
$$

We also have a specific "tail mode" energy region picked by:

$$
\omega_{K}^{2}\left(k_{H}\right) \equiv H_{0}^{2}
$$

to obtain $k_{H}$. We then have an energy calculation for the "tail" modes: 


$$
\left\langle\rho_{\text {TAIL }}\right\rangle_{K}=\frac{1}{2 \cdot \pi^{2}} \cdot \int_{K_{H}}^{\infty} k \mathrm{~d} k \int \omega_{K}(k) \cdot \mathrm{d} \omega_{K} \cdot\left|\beta_{k}\right|^{2}
$$

which is about 122 orders of magnitude smaller than

$$
\left\langle\rho_{\text {TOTAL }}\right\rangle_{K}=\frac{1}{2 \cdot \pi^{2}} \cdot \int_{0}^{\infty} k \mathrm{~d} k \int \omega_{K}(k) \cdot \mathrm{d} \omega_{K} \cdot\left|\beta_{k}\right|^{2}
$$

allowing us to write

$$
\frac{\left\langle\rho_{\text {TAIL }}\right\rangle_{K}}{\left\langle\rho_{\text {TOTAL }}\right\rangle_{K}} \approx \frac{k_{H}^{2}}{M_{P}^{4}} \cdot \omega_{K}^{2}\left(k_{H}\right) \approx \frac{H_{0}^{2}}{M_{P}^{2}} \approx 10^{-122}
$$

Here, the tail modes (of energy) are chosen as "frozen" during any expansion of the universe. This is for energy modes for frequency regions $\omega_{K}^{2}(k) \leq H_{0}^{2}$ so that we have resulting 'tail modes' of energy obeying Equation (5) above.

\section{Forming a Dispersion Relationship from Magueijo and Smolins Energy Values and Then Subsequently Modifying It}

We shall next determine what sort of dispersion relationship we can obtain by the revision of special relativity Magueijo [3] proposed. Magueijo [3] states that the energy of an independent particle will not exceed $E_{P}$ in value, which is the Planck energy. This Planck energy is the inverse of the Planck length defined by $l_{P}=\sqrt{\hbar \cdot G / c^{3}} \approx 10^{-44} \mathrm{~cm}$, where $G$ is the gravitational constant and $c$ is the speed of light. Specifically, Magueijo and Smolin [3] state that $E_{\text {PARTICLE }}=E_{P}$ if and only if the rest mass of a particle obtains an infinite value. If we set $\hbar=c=1$, we have $\left[M=M_{P}\right]=\left[E_{P}\right]$ as an upper bound. This upper bound with respect to particle energy is consistent with respect to four principles elucidated by Magueijo and Smolin [3], which are as follows:

1) Assume relativity of inertial frames: When gravitational effects can be neglected, all observers in free, inertial motions are equivalent. This means that there is no preferred state of motion.

2) Assume an equivalence principle: Under the effect of gravity, freely falling observers are all equivalent to each other and are equivalent to inertial observers.

3) A new principle is introduced: The observer independence of Planck energy. i.e. that there exists an invariant energy scale which we shall take to be the Planck energy.

4) There exists a correspondence principle: At energy scales much smaller than $E_{P}$, conventional special and general relativity are true: that is that they hold to first order in the ratio of energy scales to $E_{P}$. We ask now how can these principles be fashioned into predictions as to energy values, which we shall use to obtain dispersion relationships. Magueijo and Smolin [3] obtained a modified relationship between energy and mass:

$$
E_{0}=\frac{m_{0} \cdot c^{2}}{1+\frac{m_{0} \cdot c^{2}}{E_{P}}}
$$


which if $m=\gamma \cdot m_{0}$ and $c$ set $=1$ becomes:

$$
E=\frac{m}{1+\frac{m}{E_{P}}}
$$

We found it useful to work with, instead:

$$
E=\frac{m}{\left(1+\beta \cdot \frac{m}{E_{P}}\right)^{11}}\left(1-\frac{m}{E_{P}}\right)
$$

with a power of 11 put in the denominator due to string theory dimensions which gives us preferred numerical values we are seeking for the ratio of dark energy over total cosmological energy. If $E_{\text {PARTICLE }}<E_{P}$ and $m=\alpha \cdot k$, then $\frac{m}{E_{P}} \equiv \frac{k}{k_{P}}<1$ permits are write of Equation (9) above as (if $\beta \equiv 1000$ ):

$$
\omega_{M}(k)=\frac{\alpha \cdot k}{\left(1+\beta \frac{k}{k_{P}}\right)^{11}} \cdot\left(1-\frac{k}{k_{P}}\right)
$$

where we used $\hbar=c=1$ and $[E]=[\hbar \cdot \omega]=\left[\omega_{K}(k)\right]$ which if $k \ll k_{P}$ will lead to the same result as spoken of with the modified Epstein function [2], assuming that $|\alpha|^{2} \cong 1$, so:

$$
\omega_{M}^{2}(k) \approx k^{2}
$$

Furthermore, if $k \rightarrow k_{P}-\varepsilon_{+}$, Equation (10) will give us

$$
\omega_{M}^{2}\left(k_{P}-\varepsilon_{+}\right) \cong \varepsilon_{+}
$$

which if $\omega_{1}(k) \equiv \omega_{M}(k)$ gives the values seen in Figure 1 below.

Note how the cut off value of momentum $k_{P}$ is due to $\left(1-\frac{k}{k_{P}}\right)$ as a quantity in dispersion behavior leads to the results seen in Figure 1.

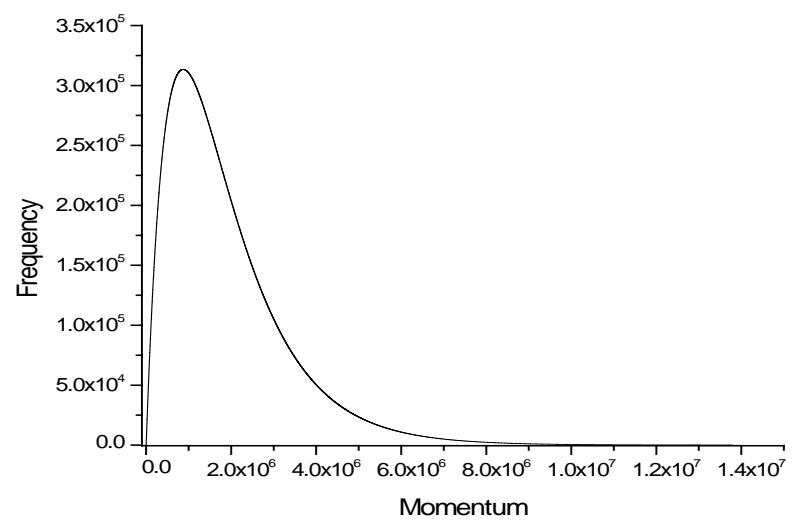

Figure 1. Graph of $1^{\text {st }}$ dispersion relationship $\omega_{M}(k)$ against momentum. This gives the desired behavior in line with the Trans Planckian dark energy hypothesis. However, $\beta \equiv 10^{3}$ ! 
We can contrast this dispersion behavior with:

$$
\omega_{1}(k)=\frac{\alpha \cdot k}{\left(1+\beta_{1} \frac{k}{k_{P}}\right)^{11}} \cdot \exp \left(-\beta_{2} \cdot \frac{k}{k_{P}}\right)
$$

We set $\beta_{1} \equiv 1$ and $\beta_{2} \equiv 100$, leading to Figure 2 as given below. Note, if $\beta_{1} \equiv 1000$ and $\beta_{2} \equiv 0$ we recover Equation (9).

So we used a tail mode energy expressions as given by

$$
\left\langle\rho_{\text {TAIL }}\right\rangle_{M}=\frac{1}{2 \cdot \pi^{2}} \cdot \int_{K_{H}}^{K_{P}} k \mathrm{~d} k \int \omega_{M}(k) \cdot \mathrm{d} \omega_{M} \cdot\left|\beta_{k}\right|^{2}
$$

and

$$
\left\langle\rho_{\text {TOTAL }}\right\rangle_{M}=\frac{1}{2 \cdot \pi^{2}} \int_{0}^{K_{P}} k \mathrm{~d} k \int \omega_{M}(k) \cdot \mathrm{d} \omega_{M} \cdot\left|\beta_{k}\right|^{2}
$$

so we obtain [2] a "frozen" tail mode energy vs. total energy ratio of

$$
\frac{\left\langle\rho_{\text {TAIL }}\right\rangle_{M}}{\left\langle\rho_{\text {TOTAL }}\right\rangle_{M}}=\frac{\int_{K_{H}}^{K_{P}} k \mathrm{~d} k \int \omega_{M}(k) \cdot \mathrm{d} \omega_{M} \cdot\left|\beta_{k}\right|^{2}}{\int_{0}^{K_{P}} k \mathrm{~d} k \int \omega_{M}(k) \cdot \mathrm{d} \omega_{M} \cdot\left|\beta_{k}\right|^{2}}<10^{-30} \text { and } \neq 10^{-122}
$$

when we are using $k_{H} \leq \frac{k_{P}}{2}$. Equation (16) has a lower bound $\approx 10^{-122}$ as stated by Mersini [2] in Equation (6) if we use $\omega_{M}\left(k_{H}\right) \approx H_{0}$. Detuning the sensitivity of this ratio to exact $k_{H} \leq(M) \cdot k_{P}$ for any $M<1$ is extremely important to the viability of our physical theory about how dark matter plays a role in inflationary cosmology.

\section{The Bogoliubov Function Used in This Paper}

We followed Mercinis [4] assumption of negligible deviations from a strictly

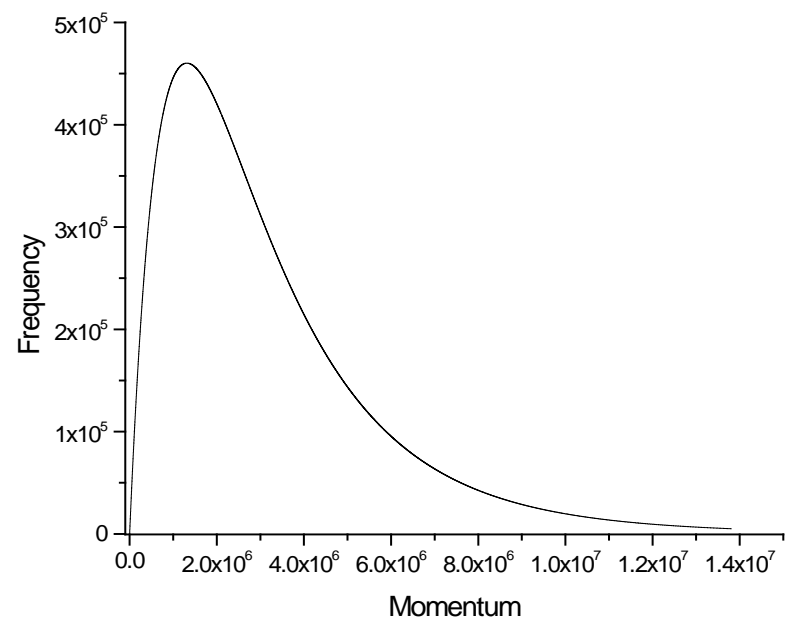

Figure 2. Graph of $2^{\text {nd }}$ dispersion relationship $\omega_{1}(k)$ against momentum which has too broad a width to be useful. 
thermal universe, and we proved it in our bogoliubov coefficient calculation. This lead to us picking the "thermality coefficient" [4] $B$ to be quite small. In addition, the ratio of confocal times as given by $\left|\frac{\eta}{\eta_{C}}\right|$ had little impact upon Equation (16). Also, $x_{0}=\frac{k}{k_{P}} \leq 1$. Therefore,

$$
\left|\beta_{k}\right|^{2} \equiv \frac{\sinh ^{2}\left(\pi \cdot \frac{B}{2} \cdot \frac{1}{k} \cdot\left|\frac{\eta}{\eta_{C}}\right|\right)+\cos ^{2}\left(\frac{\pi}{2} \cdot \sqrt{1-4 \cdot B \cdot \mathrm{e}^{-X_{O}}}\right)}{\sinh ^{2}\left(\pi \cdot(2-B) \cdot \frac{1}{k}\left|\frac{\eta}{\eta_{C}}\right|\right)-\sinh ^{2}\left(\pi \cdot \frac{B}{2} \cdot \frac{1}{k} \cdot\left|\frac{\eta}{\eta_{C}}\right|\right)}
$$

We derive this expression in the $1^{\text {st }}$ appendix entry. In addition, we note that Bastero-Gil, in 2008 in the IDM conference, of 2008 in Stockholm, brought up a discussion of the results of [6], as useful research results, and then adopted using the results of the document given in [6]. Then, Bastero-Gill when using the results of [6] subsequently delineated the size of tail energy density from Dark matter as $\rho_{X} \approx 10^{-122} M_{P}^{4}$ which is consistent with our findings that our Bogoliubov function as given by Equation (17) may be often approximated by a constant with small effects on calculating the ratio of energy for the tail vs. total energy [2] given in Equation (6) above.

\section{Analytical and Numerical Evaluation of Equation (16)}

We evaluate $\omega_{M}(k) \cdot \mathrm{d} \omega_{M}(k)$ in light of Equation (12) in our Equation (16) integrand. We then obtain:

$\omega_{M}(k) \cdot \mathrm{d} \omega_{M}(k)=\left[\frac{k \cdot\left(1-\frac{k}{k_{P}}\right)}{\left(1+\beta \frac{k}{k_{P}}\right)^{22}}-\frac{k^{2}}{k_{P}} \cdot \frac{\left(1-\frac{k}{k_{P}}\right)}{\left(1+\beta \frac{k}{k_{P}}\right)^{22}}-11 \cdot \frac{k^{2}}{k_{P}} \frac{\left(1-\frac{k}{k_{P}}\right)^{2}}{\left(1+\beta \frac{k}{k_{P}}\right)^{23}}\right] \cdot \mathrm{d} k$

and set up a numerical parameterization of

$$
\int_{A}^{K_{P}} k \mathrm{~d} k \int \omega_{M}(k) \cdot \mathrm{d} \omega_{M} \cdot\left|\beta_{k}\right|^{2}
$$

with $\beta_{k}$ chosen by considerations presented in Mercini's [4] $2^{\text {nd }}$ paper.

\section{Why We Still Were Unable to Match Cosmic Ray Data and Found Our Dispersion Relationship Not Physically \\ Tenable}

$\beta \equiv 1000$ in Equation (10) was picked so $k_{H}$ could have a wide range of values. This permitted $\frac{\left\langle\rho_{\text {TAIL }}\right\rangle_{M}}{\left\langle\rho_{\text {TOTAL }}\right\rangle_{M}}$ to be bounded below by a value $\leq 10^{-30}$ for $k_{H} \leq \frac{k_{P}}{2}$ in line with de tuning the sensitivity of the ratio results if we use $\beta \equiv 1000$ in the Equation (10) dispersion relationship. We obtain Mercini's main result [2] at the expense of not matching cosmic ray data [1]. We should note that Equation (13) lead to a far broader dispersion curve width as given in 
Figure 2, which also necessitated a far larger $k_{H}$ value needed to have the frequency $\omega_{M}\left(k_{H}\right) \approx H_{0}$ as used by Mercini [2]. This in turn leads to a much bigger value for a lower bound for Equation (16) than what would obtain numerically if we used Equation (10) for dispersion. Detuning the sensitivity of this ratio to be $k_{H} \leq(M) \cdot k_{P}$ for any $M<1$ is extremely important to the viability of our physical theory about how dark matter plays a role in inflationary cosmology. We find that this result is still not sufficient to match the cosmic ray problem [1] since Equation (10) gives us:

$$
\omega_{M}(k) \underset{K \ll K_{P}}{\longrightarrow} \frac{k}{\left(1+\beta_{3} \frac{k}{k_{P}}\right)}
$$

The $\beta_{3} \cong 11 \times 10^{+3}$ whereas we would prefer to find $\beta_{3} \cong 11 \times 10^{-10}$.

\section{Can $\beta_{3} \cong 11 \times 10^{-10}$ with a Modified Dispersion Relationship?}

The answer is no even after a modification of our dispersion relationship:

$$
\omega_{2}(k)=\frac{\alpha \cdot k}{\left(1+\beta\left(\frac{k}{k_{P}}\right)^{L}\right)^{11}} \cdot\left(1-\left(\frac{k}{k_{P}}\right)^{L}\right)
$$

With $L=2$, then 3 put in. However, even with a value of $L=2$ put in Equation (21) we obtained, for $\beta \equiv 2.25$ and $k_{H} \equiv \frac{k_{P}}{2}$

$$
\frac{\left\langle\rho_{\text {TAIL }}\right\rangle_{2}}{\left\langle\rho_{\text {TOTAL }}\right\rangle_{2}}=\frac{\int_{K_{H}}^{K_{P}} k \mathrm{~d} k \int \omega_{2}(k) \cdot \mathrm{d} \omega_{2} \cdot\left|\beta_{k}\right|^{2}}{\int_{0}^{K_{P}} k \mathrm{~d} k \int \omega_{2}(k) \cdot \mathrm{d} \omega_{2} \cdot\left|\beta_{k}\right|^{2}} \leq 6.425 \times 10^{-3}
$$

which has a very different lower bound than the behavior seen in Equation (16). If we pick $\beta \equiv 10^{-10}$ as suggested by T. Jacobson [1] to try to "solve" the cosmic ray problem, we then find that Equation (22) approaches unity which thereby throws into question the Trans-Planckian dark energy hypothesis. Indeed, we believe that the entire Trans-Plankian model of Dark energy makes initial conditions, which contravene known astrophysical cosmic ray data [1] that has been collected in the last decade. Graphically, having even $\beta \equiv 2.25$ for Equation (21) in Figure 3 creates a dispersion versus momentum graph, which is much greater in width than Figure 1 which has a much larger $\beta \equiv 10^{3}$ value. Appendix entry 2 shows us that we still could not match the beta coefficient values [1] needed to solve the cosmic ray problem of special relativity.

\section{Conclusion}

We found that the dispersion relationship given in Equation (10) and its limiting behavior shown in Equation (20) gives the lower bound behavior as noted in Equation (16) above for a wide range of possible $k_{H} \leq M \cdot k_{P}$ values if $M<1$ 


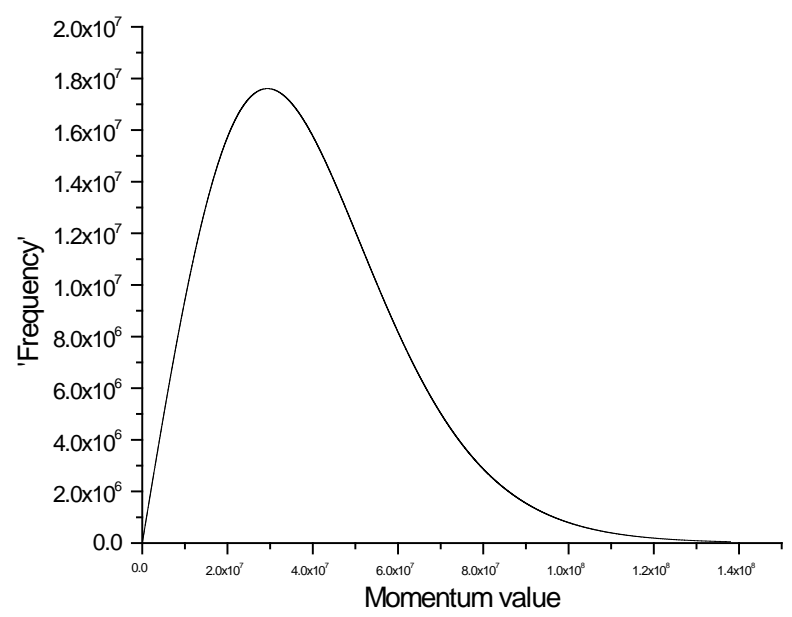

Figure 3. Graph of $3^{\text {rd }}$ dispersion relationship $\omega_{2}(k)$ against momentum which is still too broad in width, and has $\beta \equiv 2.25$.

above. This was, however, done for a physically unacceptably large $\beta \equiv 10^{3}$ value [1] while we wanted, instead $\beta \equiv 10^{-10}$ in order to solve the cosmic ray problem [1]. Our additional modifications of dispersion relationships as noted in Appendix 2 still lead to unacceptably large dark energy versus total energy values. We then conclude that the Trans Planckian dark energy hypothesis contravenes known solutions to the cosmic ray problem of special relativity and is thereby in need of substantial revision. And we think that this document should be compared against the predictions given in [7], since modification of the Dark Energy hypothesis may impact gravity theories. Their confirmation or rejection will be affecting predictions done by Corda, and require careful analysis, and are integral to a fuller understanding of scalar-tensor gravitational alternatives to General Relativity.

\section{Acknowledgements}

This work is supported in part by National Nature Science Foundation of China grant No. 110752.

\section{References}

[1] Jacobson, T., Liberati, S. and Mattingly, D. (2003) Threshold Effects and Planck Scale Lorentz Violations: Combined Constraints from High Energy Astrophysics. Physical Review D, 67, 124011. https://doi.org/10.1103/PhysRevD.67.124011

[2] Mersini, L., Bastero-Gil, M. and Kanti, P. (2001) Relic Dark Energy from the Trans-Planckian Regime. Physical Review D, 64, 043508. https://doi.org/10.1103/PhysRevD.64.043508

[3] Magueijo, J. and Smolin, L. (2002) Lorenz Invariance with an Invariant Energy Scale. Physical Review Letters, 88, 190403. https://doi.org/10.1103/PhysRevLett.88.190403

[4] Bastero-Gil and Mersini. (2001) SN1a data and the CMP of Modified Curvature at Short and Large Distances. Physical Review D, 65, 023502. https://doi.org/10.1103/PhysRevD.65.023502 
[5] Lemoine, M., Martin, J. and Uzan, J.-P. (2003) Trans-Planckian Dark Energy? Physical Review D, 67, 103520. https://doi.org/10.1103/PhysRevD.67.103520

[6] Moulin, E., Mayet, F., D. and Santos, D. (2005) Supersymmetric Dark Matter Search via Spin-Dependent Interaction with 3He. Physics Letters B, 614, 143-154.

https://arxiv.org/abs/astro-ph/0503436v1 https://doi.org/10.1016/j.physletb.2005.03.068

[7] Corda, C. (2009) Interferometric Detection of Gravitational Waves: The Definitive Test for General Relativity. International Journal of Modern Physics D, 18, 2275-2282. https://arxiv.org/abs/0905.2502 https://doi.org/10.1142/S0218271809015904 


\section{Appendix Entry 1: Deriving the Bogoliubov Coefficient for Section III}

\section{Part I. Initial Assumptions}

We derive the Bogoliubov coefficient, which is used in Equation (16) of the main text. We refer to Mersini's article [4] which has a Bogoliubov coefficient which takes into account a deviation function $\Gamma\left(k_{0}, B\right)$, which is a measure of deviation from thermality [4] in the spectrum of co moving frequency values $\Omega_{n}(k)$ over different momentum values. Note that $\eta$ is part of a scale factor $a(\eta)=\left|\eta_{C} / \eta\right|$ and $k=n / a(\eta)$ so that "momentum" $k \propto|\eta|$. Also if we are working with the conformal case of $\varepsilon=1 / 6$ appearing [4] in:

$$
\Omega_{n}^{2}=a^{2}(\eta) \cdot \omega_{N O N-L I N}^{2}(k)-(1-6 \cdot \varepsilon) \cdot \frac{a^{\prime \prime}}{a}=a^{2}(\eta) \cdot \omega_{N O N-L I N}^{2}(k)=a^{2}(\eta) \cdot F^{2}(k)(1)
$$

then for small momentum:

$$
\omega_{N O N-L I N}^{2}\left(\tilde{k}_{0}\right) \approx \tilde{k}_{0}^{2}
$$

if "momentum" $\tilde{k}_{0} \ll k_{P}$, where we use the same sort of linear approximation used by Mercini [2], as specified for Equation (17) of their article [2] if the Epstein function specified in Equation (1) of the main text has a linear relationship. We write out a full treatment of the dispersion function $F(k)$ [4] since it permits a clean derivation of the Bogoliubov coefficient which has the deviation function $\Gamma\left(k_{0}, B\right)$. We begin with [4]:

$$
\left|\beta_{k}\right|^{2} \equiv\left|\beta_{n}\right|^{2}=\frac{\sinh ^{2}\left(2 \cdot \pi \cdot \hat{\Omega}_{-}\right)+\Gamma\left(k_{0}, B\right)}{\sinh ^{2}\left(2 \cdot \pi \cdot \hat{\Omega}_{+}\right)-\sinh ^{2}\left(2 \cdot \pi \cdot \hat{\Omega}_{-}\right)}
$$

where we get an appropriate value for the deviation function $\Gamma\left(k_{0}, B\right)$ [4] based upon having the square of the dispersion function $F(k)$ obey Equations (1) and (2) above for $\tilde{k}_{0} \ll k_{P}$. Note, $k_{P}$ is a maximum momentum value along the lines Magueijo [3] suggested for an $E_{P}$ Plank energy value.

\section{Part II. Deriving Appropriate $\Gamma\left(k_{0}, B\right)$ Deviation Function Val-} ues

We look at how Bastero-Gil [4] obtained an appropriate $\Gamma\left(k_{0}, B\right)$ value. Basterero-Gil wrote:

$$
\Gamma\left(k_{0}, B\right)=\cosh ^{2}\left(\frac{\pi}{2} \cdot \sqrt{4 \cdot B \cdot \mathrm{e}^{-X_{o}}-1}\right)
$$

with

$$
x_{0}=\frac{\tilde{k}_{0}}{k_{P}} \ll 1
$$

and

$$
F^{2}(k)=\left(k^{2}-\tilde{k}_{1}^{2}\right) \cdot V_{0}\left(x, x_{0}\right)+k^{2} \cdot V_{1}\left(x-x_{0}\right)+\tilde{k}_{1}^{2}
$$


where $\tilde{k}_{1}<k_{P}$ and where $\tilde{k}_{1}$ is in the Trans-Planckian regime but is much greater than $k_{0}$. We are determining what $B$ should be in Equation (16) of the main text provided that $F(k) \approx k$ as $x=\frac{\tilde{k}}{k_{P}} \rightarrow x_{0}$ which will lead to specific restraints we place upon $V_{0}\left(x, x_{0}\right)$ as well as $V_{1}\left(x-x_{0}\right)$ above. Following Bastero-Gil [4], we write:

$$
V_{0}\left(x, x_{0}\right)=\frac{C}{1+\mathrm{e}^{X}}+\frac{E \cdot \mathrm{e}^{X}}{\left(1+\mathrm{e}^{X}\right) \cdot\left(1+\mathrm{e}^{X-X_{O}}\right)}
$$

and:

$$
V_{1}\left(x-x_{0}\right)=-B \cdot \frac{\mathrm{e}^{X}}{\left(1+\mathrm{e}^{X-X_{O}}\right)^{2}}
$$

When $x=\frac{\tilde{k}}{k_{P}} \rightarrow x_{0} \ll 1$ we get [2] [4]

$$
F^{2}\left(k_{0}\right) \equiv \omega_{N O N-L I N}^{2}\left(k_{0}\right) \cong-k_{1}^{2} \cdot\left(1-\frac{c}{2}-\frac{E}{4}\right)+k_{0}^{2} \cdot\left(\frac{c}{2}+\frac{E}{4}-\frac{B}{4}\right) \cong k_{0}^{2}
$$

which then implies $0<B \approx \varepsilon_{+} \ll 1$. Then we obtain:

$$
\Gamma\left(k_{0}, B \cong \varepsilon_{+}\right) \cong \cosh ^{2}\left(\left(\frac{\pi}{2}+\varepsilon_{+}\right) \cdot i\right) \approx \varepsilon_{+} \ll 1
$$

and

$$
\left|\beta_{k}\right|^{2} \equiv\left|\beta_{n}\right|^{2} \cong \frac{\sinh ^{2}\left(2 \cdot \pi \cdot \hat{\Omega}_{-}\right)+\varepsilon_{+}}{\sinh ^{2}\left(2 \cdot \pi \cdot \hat{\Omega}_{+}\right)-\sinh ^{2}\left(2 \cdot \pi \cdot \hat{\Omega}_{-}\right)}
$$

\section{Part III. Finding Appropriate $\hat{\Omega}_{+}$and $\hat{\Omega}_{-}$Values}

We define, following Bastero-Gil [4]

$$
\hat{\Omega}_{ \pm}=\frac{1}{2} \cdot\left(\hat{\Omega}_{\text {OUT }} \pm \hat{\Omega}_{I N}\right)
$$

where we have that

$$
\Omega^{\text {OUT }}=\stackrel{\eta \rightarrow \infty}{\longrightarrow} \Omega_{n}(\eta \equiv \infty)
$$

and

$$
\Omega^{I N}=\stackrel{\eta \rightarrow-\infty}{\longrightarrow} \Omega_{n}(\eta \equiv-\infty)
$$

whereas we have that

$$
\hat{\Omega}_{\tilde{k}}=\frac{\Omega_{\tilde{k}}}{n}
$$

where $\tilde{k}$ denotes either out or in. Also:

$$
\Omega^{\text {OUT }} \cong \Omega^{I N} \cong 1
$$

which lead to: 


$$
\hat{\Omega}_{+} \cong\left(1-\frac{B}{2}\right) \cdot \frac{1}{n}=\left(1-\frac{B}{2}\right) \cdot \frac{1}{k} \cdot\left|\frac{\eta}{\eta_{C}}\right| \cong \frac{1}{k} \cdot\left|\frac{\eta}{\eta_{C}}\right|
$$

as well as

$$
\hat{\Omega}_{-} \cong \frac{B}{2} \cdot \frac{1}{n} \cong 0
$$

\section{Appendix Entry 2: How Equation (16) of Text Changes for Varying $\beta$ Values and Different Dispersion Relationships}

Starting with Equation (21) of the main text.

If $\beta=1.05$ and $L=1 / 2,\left(\frac{k}{k_{P}}\right) \rightarrow \sqrt{\frac{k}{k_{P}}}$, then $\frac{\left\langle\rho_{\text {TAIL }}\right\rangle_{M}}{\left\langle\rho_{\text {TOTAL }}\right\rangle_{M}} \cong 0.371$

If $\beta=1.05$ and $L=1,\left(\frac{k}{k_{P}}\right) \rightarrow\left(\frac{k}{k_{P}}\right)$, then $\frac{\left\langle\rho_{\text {TALL }}\right\rangle_{M}}{\left\langle\rho_{\text {TOTAL }}\right\rangle_{M}} \cong 0.263$

If $\beta=1.05$ and $L=2,\left(\frac{k}{k_{P}}\right) \rightarrow\left(\frac{k}{k_{P}}\right)^{2}$, then $\frac{\left\langle\rho_{\text {TAIL }}\right\rangle_{M}}{\left\langle\rho_{\text {TOTAL }}\right\rangle_{M}} \cong 0.115$

If $\beta=10.5$ and $L=1 / 2,\left(\frac{k}{k_{P}}\right) \rightarrow \sqrt{\frac{k}{k_{P}}}$, then $\frac{\left\langle\rho_{\text {TAIL }}\right\rangle_{M}}{\left\langle\rho_{\text {TOTAL }}\right\rangle_{M}} \cong 1.935 \times 10^{-5}$

If $\beta=10.5$ and $L=1,\left(\frac{k}{k_{P}}\right) \rightarrow\left(\frac{k}{k_{P}}\right)$, then $\frac{\left\langle\rho_{\text {TALL }}\right\rangle_{M}}{\left\langle\rho_{\text {TOTAL }}\right\rangle_{M}} \cong 7.347 \times 10^{-6}$

If $\beta=10.5$ and $L=2,\left(\frac{k}{k_{P}}\right) \rightarrow\left(\frac{k}{k_{P}}\right)^{2}$, then $\frac{\left\langle\rho_{\text {TAIL }}\right\rangle_{M}}{\left\langle\rho_{\text {TOTAL }}\right\rangle_{M}} \cong 6.7448 \times 10^{-8}$

We need $\beta \cong 10^{-10}$ with $\frac{\left\langle\rho_{\text {TAIL }}\right\rangle_{M}}{\left\langle\rho_{\text {TOTAL }}\right\rangle_{M}} \leq 10^{-30}$ to get our results via this

Trans-Plankian model to be consistent with physically verifiable solutions to the cosmic ray problem. 